\title{
Laboratory strategy for autoantibodies testing as a diagnostic marker of pulmonary fibrosis in systemic sclerosis: A preliminary study prior to cohort registry of systemic sclerosis in West Java Indonesia
}

\author{
Anna Tjandrawati ${ }^{1 \oplus}$, Verina Logito ${ }^{1, *}{ }^{\oplus}$, Winda Agnestia Maranna Saragih ${ }^{2}$, Andri Reza Rahmadi ${ }^{2}$, \\ Sumartini Dewi ${ }^{2}$
}

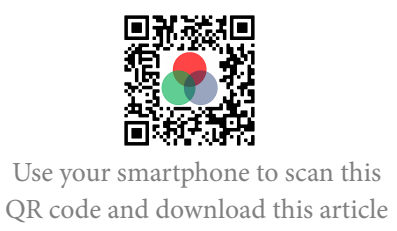

${ }^{1}$ Department of Clinical Pathology, Faculty of Medicine Universitas Padjadjaran/ Dr. Hasan Sadikin General Hospital Bandung, Indonesia

${ }^{2}$ Department of Internal Medicine, Faculty of Medicine Universitas Padjadjaran/ Dr. Hasan Sadikin General Hospital Bandung, Indonesia

Correspondence

Verina Logito, Department of Clinical Pathology, Faculty of Medicine Universitas Padjadjaran/ Dr. Hasan Sadikin General Hospital Bandung, Indonesia

Email: verinalogito@gmail.com

History

- Received: Apr 27, 2021

- Accepted: Aug 03, 2021

- Published: Aug 29, 2021

DOI : 10.15419/bmrat.v8i8.689

\section{Check for updates}

Copyright

(c) Biomedpress. This is an openaccess article distributed under the terms of the Creative Commons Attribution 4.0 International license.

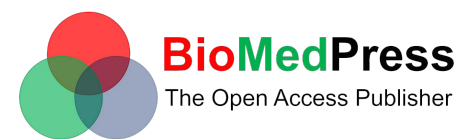

\begin{abstract}
Background: Interstitial lung disease (ILD) is a complication often found in patients with Systemic Sclerosis (SSc). This usually manifests as pulmonary fibrosis and is the leading cause of death in SSc patients. Antinuclear antibodies indirect immunofluorescence (ANA-IIF) test and specific autoantibodies testing could be employed as a diagnostic marker to detect pulmonary fibrosis, but the high cost of these tests often burdens patients in developing countries. This study aims to compare ANA-IIF and an alternative, possibly inexpensive method to detect pulmonary fibrosis in SSc. Methods: This is a preliminary study prior to the cohort systemic sclerosis registry conducted in Rheumatology Outpatient Clinic and Clinical Pathology Department at Dr. Hasan Sadikin General Hospital, Bandung, from January 1, 2018 to December 31, 2020. Two contrastive laboratory testing strategies were followed to confirm pulmonary fibrosis in 61 SSc patients. The first strategy was to perform antinuclear antibodies indirect immunofluorescence (ANA-IIF) test followed by specific autoantibodies detection [anti-topoisomerase-I (ATA), Anti-Th/To, SSc Profile, Immunoblot]. The alternative strategy included conducting the tests for SSc autoantibodies in the reverse order. Results: A total of 42 out of 61 (68.8\%) subjects were diagnosed with pulmonary fibrosis by high-resolution computed tomography (HRCT) scan. The first strategy detected dominant specific pulmonary fibrosis autoantibodies in subjects with pulmonary fibrosis: homogeneous nucleolar pattern was dominant ( $n=25,59.52 \%)$, followed by nucleolar pattern $(n=17,40.48 \%)$. Of all the subjects with a homogeneous nucleolar pattern, ATA was detected in 15 subjects (60\%), the combination of ATA and Th/To in 9 subjects (36\%), and Ro52 in one subject (4\%). The second strategy yielded positive autoantibodies results in 29 out of 42 (69.05\%) subjects, ATA in 20 subjects (47.61\%), and Th/To in 9 patients (21.44\%), but failed to detect any autoantibodies specific to SSc-ILD in 13 subjects (30.95\%). Conclusion: The first strategy [ANA-IIF followed specific autoantibodies testing (ATA, Th/To, SSc profile and immunoblot)] is more likely to detect specific autoantibodies toward ILD in SSc patients.
\end{abstract}

Key words: ANA-IIF, autoantibodies screening, pulmonary fibrosis, SSC profile, systemic sclerosis

\section{INTRODUCTION}

Systemic sclerosis (SSc) is a complex systemic autoimmune rheumatic disease with complicated pathophysiology and heterogeneity of organ involvements ${ }^{1}$. The cause is yet to be determined. It was suggested that both genetic and environmental factors should be considered. The hallmark of SSc pathogenesis is endothelial cell injuries, followed by aberrant vascular and production of autoantibodies. Excessive deposition and accumulation of extracellular matrix are common in these conditions. As a consequence of radical tissue remodeling, tissue architecture may be destroyed and organ function may be lost ${ }^{2}$. The clinical manifestations of SSc depend on the organs in- volved, such as the skin, lungs, heart, kidneys, and gastrointestinal tract.

Interstitial lung disease (ILD) is commonly found in SSc and leads to pulmonary fibrosis. Interstitial lung disease affects the tissue and space surrounding the lungs' air sacs ${ }^{3}$. The incidence of SSc with pulmonary manifestations in 2016 in Europe was four in 100,000 individuals per year. In Europe, approximately $35 \%$ of SSc patients have pulmonary manifestations ${ }^{4,5}$. However, in Dr. Hasan Sadikin (RSHS) Hospital Bandung, Indonesia, there are no details on the occurrence of SSc with and without pulmonary manifestations.

Cite this article : Tjandrawati A, Logito V, Saragih W A M, Rahmadi A R, Dewi S. Laboratory strategy for autoantibodies testing as a diagnostic marker of pulmonary fibrosis in systemic sclerosis: A preliminary study prior to cohort registry of systemic sclerosis in West Java Indonesia. Biomed. Res. Ther.; 8(8):4505-4513. 
The current diagnosis of SSc was made using the criteria developed by The American College of Rheumatology/European League against Rheumatism (ACR/EULAR) in 2013, which included laboratory tests (detection of specific autoantibodies) ${ }^{1,4}$. Patients with SSc have specific autoantibodies, such as anti-centromere, anti-topoisomerase I, anti-RNA polymerase III, and several other autoantibodies ${ }^{6}$. Clinical symptoms (skin thickness, changes in organ involvement) and laboratory parameters that characterize inflammatory activity, fibrosis, and vasculopathy are used to assess the disease activity in $\mathrm{SSc}^{2}$.

Systemic Sclerosis-related Interstitial Lung Disease (SSc-ILD) is a severe SSc manifestation that can lead to death ${ }^{7}$. The presence of ILD in SSc patients influences the disease's severity and prognosis ${ }^{3}$. HighResolution Computed Tomography (HRCT) of the chest is the gold standard and evaluation of SSc specific autoantibodies: anti-topoisomerase I (ATA) and anti-Th/ToRNP are the two factors to consider when diagnosing SSc with pulmonary manifestations ${ }^{8,9}$. The pathogenesis of this disease is complicated and is still unknown. Autoantibodies, on the other hand, are a serologic hallmark of the disorder and are useful as diagnostic and prognostic biomarkers. Autoantibodies directed against one or more autoantigens are found in up to $95 \%$ of SSc patients ${ }^{10}$.

Although there is no current algorithm for SSc-ILDspecific autoantibody monitoring, specific autoantibodies detection plays an important role as a diagnostic marker for pulmonary fibrosis ${ }^{9}$. The first strategy is commonly used in diagnosing SSc-ILD. The first strategy begins with a screening examination with ANA-IIF followed by a specific autoantibodies examination, namely the SSc profile. Thoracic HRCT examination is the gold standard in diagnosing SSc-ILD. The problem faced in this radiological examination is that not all health care organizations possess HRCT facilities, and they are often restricted to big cities. The test of specific autoantibodies (first strategy) is expected to overcome this limitation. In addition to being affordable, it makes examination procedures easier. Considering the price of the HRCT, IIF ANA, and SSc profile examinations which are quite expensive, the author wants to investigate whether a specific autoantibody examination can only be carried out without an autoantibody screening examination (second strategy) to detect pulmonary fibrosis. This study aimed to compare two laboratory diagnostic strategies for detecting pulmonary fibrosis in SSc.

\section{METHODS}

A preliminary study prior to cohort registry systemic sclerosis was conducted in Rheumatology Outpatient Clinic and Clinical Pathology Department at Dr. Hasan Sadikin General Hospital Bandung, from 1 January 2018 until 31 December 2020. This was a descriptive study with a cross-sectional design. The research was approved by the local Ethics Committee once the patients gave their informed consent. The American College of Rheumatology guidelines for SSc (2013) was used to make the diagnosis ${ }^{1}$.

Patients with a diagnosis of SSc who visited the RSHS Rheumatology Outpatient Clinic between January 1, 2018, and June 30, 2020, make up the study's population. The inclusion criteria for this study were patients with SSc or Mixed Connective Tissue Disease (MCTD) with ILD images on HRCT examination. The exclusion criteria for this study were patients with lung tuberculosis or a history of lung tuberculosis All the patients underwent an HRCT scan of the chest as well as a pulmonary function test (PFT) on the same day. The reference technique for the noninvasive diagnosis of ILD is an HRCT scan of the chest, which offers excellent parenchymal detail ${ }^{11}$. The patient's serum was collected for autoantibodies immunofluorescence test (IIF ANA, Euroimmun), and specific autoantibodies test (SSc profile, Immunoblot, Euroimmun).

The most commonly used technique for ANA determination is indirect immunofluorescence (IIF) ${ }^{12}$. To detect specific autoantibodies, antigens from human epithelial cell line-2 (Hep-2) cells, which are derived from human larynx carcinoma cells, are used ${ }^{13}$. The cut-off for positive test results is 1:160, but due to the high variability of the test and technician-dependent variations, this cannot be generalized. High autoantibodies titers ( $>1: 160)$ can be detected in the patients, but only $5 \%$ in healthy people ${ }^{14}$. Low autoantibody titers (1:40) can be found in $25-30 \%$ of healthy controls, and therefore should be regarded as negative test results ${ }^{15}$.

Immunoblot is the method of choice for the confirmatory test. Blot strips are used as an antigen-containing solid phase. They are coated with antigen extracts separated by electrophoresis according to the molecular mass. The Immunoblot strips are also coated with membrane chips that contain highly purified native or recombinant antigens. If the sample contains specific autoantibodies, these will bind to the membranebound antigens. In the next step, an alkaline phosphatase labeled autoantibodies (conjugate) is added, which binds to the specific autoantibodies. If specific autoantibodies are present in the patient sample, 


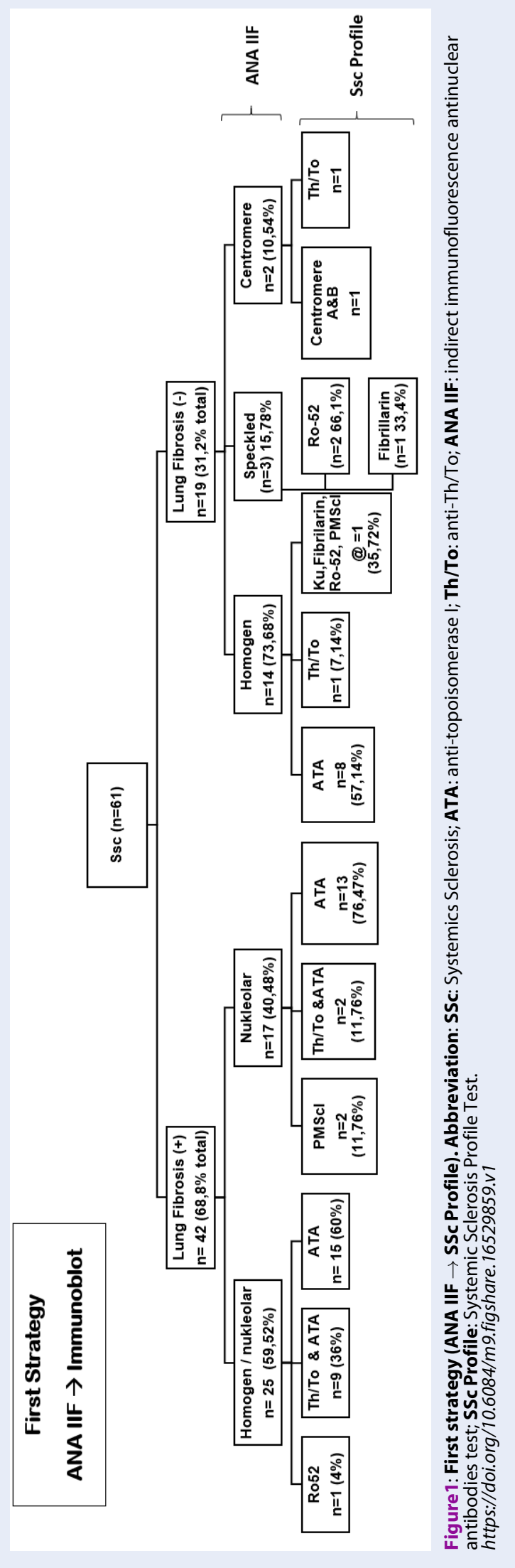




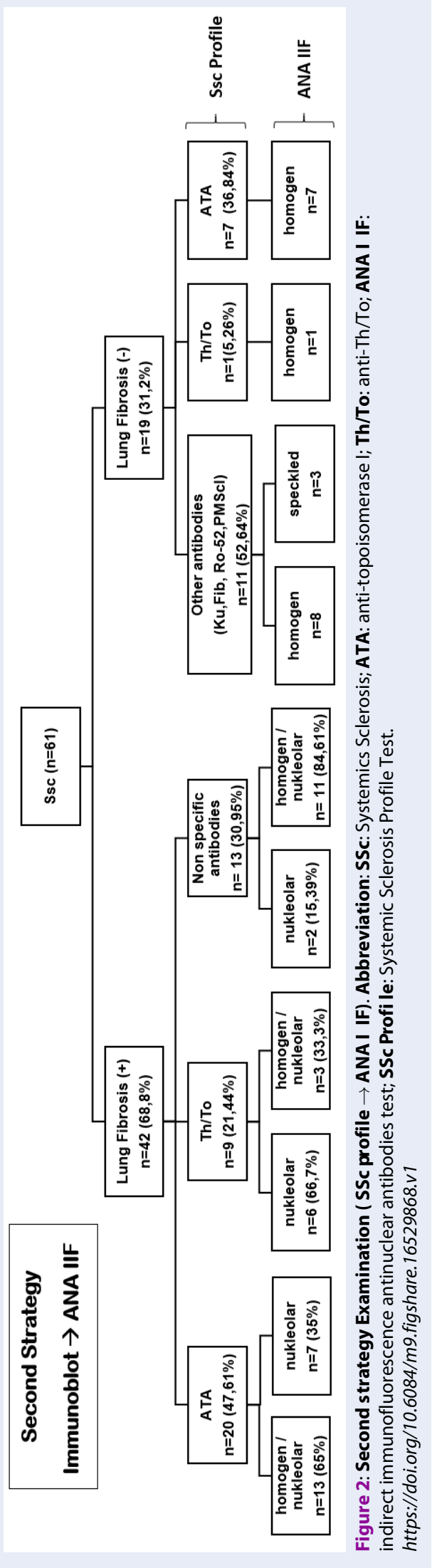


a dark line appears at the respective antigen position. The staining strength is proportional to the number of autoantibodies present in the sample. The staining of the control band at the lower end of the strip indicates that the individual incubation steps were completed correctly. The evaluation is performed automatically with the EUROLineScan software ${ }^{16}$.

Patients who met the inclusion criteria had their lungs checked for pulmonary fibrosis using a gold standard diagnostic method called HRCT. Two laboratory diagnostic strategies were described. The first strategy, which consisted of a screening test and a confirmation autoantibodies test: antinuclear autoantibodies screening (ANA IIF, Euroimmun) with specific autoantibodies detection Anti-topoisomerase I (ATA) and Anti Th/To (SSc profile, Immunoblot, Euroimmun). The second strategy was a contrastive method with the first one, which began with specific autoantibodies detection and then moved on to autoantibodies screening. The data collected were tabulated and presented in the form of descriptive summaries.

\section{RESULTS}

A total of 61 patients were included, with 42 of them having pulmonary fibrosis $(68.8 \%)$, the mean age of 42 years, and the study group had female predominance $(94.73 \%)$. There were 11 patients with a limited type of SSc, 45 patients with a diffuse type of SSc, and 5 patients with overlap syndrome, according to the clinical subtypes of the diseases.

Using the first strategy method (Figure 1), autoantibodies screening of homogenous nucleolar pattern is dominantly found ( $\mathrm{n}=25,59.52 \%)$ and in combination with ATA antibodies $(\mathrm{n}=15,60 \%)$, ATA \& Th/To antibodies $(\mathrm{n}=9,36 \%)$ in the total $42(100 \%)$ subjects with pulmonary fibrosis. Meanwhile, using the second strategy method (Figure 2), a total of 29 (69.05\%) subjects with specific pulmonary fibrosis autoantibodies were found, twenty subjects (47.61\%) of them with ATA autoantibodies, and homogenous nucleolar pattern positivity reaching $65 \%(n=13)$. Nine subjects (21.44\%) had Th/To antibodies, and 3 subjects had a combination of homogenous/nucleolar patterns $(33.3 \%)$. We found interesting results using the second strategy (Figure 2), in which there were 13 (30.95\%) patients with non-specific SSc autoantibodies. A summary of the research results can be seen in the Figures 1 and 2.

To compare the differences between the two strategies, data will be analyzed with a p-value $<0.05$. We analyzed the relationship between specific autoantibodies with lung fibrosis in the first strategy with the Chi-square test in Table 1.
From the results of the analysis using the Chi-square test, it is obtained that - the relationship between specific autoantibodies with lung fibrosis in the first strategy is $p=0.000252(p<0.05)$, which means that there is a significant relationship between specific autoantibodies with lung fibrosis in the first strategy. We analyzed the relationship between specific autoantibodies with lung fibrosis in the second strategy with the Chi-square test in Table 2.

From the results of the analysis using the Chi-square test, it is obtained that the relationship between specific autoantibodies with lung fibrosis in the second strategy is $\mathrm{p}=0.086933$ ( $\mathrm{p}>0.05)$, which means that there is no significant relationship between specific autoantibodies with lung fibrosis in the second strategy.

\section{DISCUSSION}

Systemic sclerosis is a complex autoimmune disease whose pathogenesis is characterized by three hallmarks: small vessel vasculopathy, production of autoantibodies, and fibroblast dysfunction leading to increased deposition of the extracellular matrix ${ }^{1}$. Patients with SSc often show pulmonary involvement, which most commonly manifests as fibrosis or interstitial lung disease (ILD) ${ }^{7}$. It's reported that $80 \%$ of SSc patients have pulmonary disease. Furthermore, patients with SSc who have pulmonary involvement have a relatively poor prognosis, and pulmonary disease is the leading cause of death for SSc patients ${ }^{13}$. The diagnosis of SSc was based on clinical symptoms and the presence of SSc-specific autoantibodies (the criteria of the ACR/EULAR in 2013). The American College of Rheumatology/European League against Rheumatism includes several autoantibodies that are specific for SSc disease, including ATA, anticentromere, and anti-RNA polymerase ${ }^{1}$. Laboratory examinations have a key role in diagnosing SSc. The main tests that play a role are autoantibodies examinations. The immunological examination of SSc is divided into two stages, i.e., a screening test and a follow-up/confirmation test. For SSc screening purposes, the indirect immunofluorescence anti-nuclear autoantibodies (IIF-ANA) method is used ${ }^{13}$. Postscreening, the examination of autoantibodies can be followed by highly specific autoantibodies examinations/confirmatory tests, such as the autoantibodies mono test using the ELISA method or the ANA/SSc profile ${ }^{17,18}$. Autoantibodies are a serologic hallmark of the disease and have been proven invaluable as diagnostic and prognostic biomarkers ${ }^{10}$.

Pulmonary involvement is linked to a poor prognosis in patients with SSc, so it is important to detect 
Table 1: The relationship between specific autoantibodies with lung fibrosis in the first strategy

\begin{tabular}{ccc} 
& Specific autoantibodies SSc-ILD & $\begin{array}{c}\text { Non-specific autoantibodies SSc-ILD } \\
\text { N (\%) }\end{array}$ \\
\hline Lung Fibrosis & $39(92.8 \%)$ & $3(7.2 \%)$ \\
No Lung Fibrosis & $10(52.6 \%)$ & $9(47.4 \%)$ \\
\hline
\end{tabular}

Note: Analysis with Chi-Square Test

Specific autoantibodies SSc-ILD: ATA, Th/To, ATA and Th/To

Non-specific autoantibodies SSc-ILD: Ro-52, PMScl, Ku, Fibrillarin, Centromere A\&B

Table 2: The relationship between specific autoantibodies with lung fibrosis in the second strategy

\begin{tabular}{ccc} 
& $\begin{array}{c}\text { Specific autoantibodies SSc-ILD } \\
\text { N (\%) }\end{array}$ & $\begin{array}{c}\text { Non-specific autoantibodies SSc-ILD } \\
\mathbf{N}(\%)\end{array}$ \\
\hline Lung Fibrosis & $29(69 \%)$ & $13(31 \%)$ \\
No Lung Fibrosis & $8(42.2 \%)$ & $11(57.8 \%)$ \\
\hline
\end{tabular}

Note: Analysis with Chi-Square Test

Specific autoantibodies SSc-ILD: ATA, Th/To, ATA and Th/To

Non-specific autoantibodies SSc-ILD: Ro-52, PMScl, Ku, Fibrillarin

and recommend interventions against ILD earlier ${ }^{19}$. With asymptomatic disease progression i.e., without dyspnea or cough, the diagnosis is often delayed. Up on HRCT scan, up to $90 \%$ of patients will have interstitial anomalies, and $40-75 \%$ will have pulmonary function test (PFT) changes ${ }^{7}$. Early parenchymal lung involvement is common after SSc diagnosis, with $25 \%$ of patients developing clinically significant lung disease within three years as identified by physiological and radiographic abnormalities ${ }^{7}$. Patients with the diffuse type of SSc have a higher rate of ILD than those with the limited type of SSc. The European Scleroderma Trials and Research group found that the incidence of ILD was 53\% in diffuse-type SSc and 35\% in limited-type SSc in 3,656 patients with $\mathrm{SSc}^{20}$.

We use two laboratory diagnostic strategies. In the first strategy (Figure 1), we begin with ANAIIF. There were 39 (92.85\%) subjects with pulmonary fibrosis-specific autoantibodies; autoantibodies screening was dominated by a homogeneous nucleolar pattern $(\mathrm{n}=25,59.52 \%)$ and combination with ATA were 15 patients (60\%). This homogenous nucleolar pattern can be found in several SSc-ILDspecific autoantibodies, namely ATA, and the nucleolar pattern is found in anti-Th/To RNP. Then we proceeded with Immunoblot and Euroline examination; the dominance of ATA autoantibodies was observed. This meant that the first strategy could diagnose all SSc-ILD patients. The results from this first strategy are per research from Bahmer et al. stating that the specific autoantibodies in SSc-ILD are ATA and antiTh/To ${ }^{13}$. Patients with SSc-ILD have specific autoantibodies, namely ATA and anti-Th/To RNP. Research from Solomon et al. explained that ATA found from SSc patients will increase the incidence of SSc-ILD ${ }^{7}$. Anti-topoisomerase I is found in $15-42 \%$ of SSc patients, with a specificity of $90-100 \%$. SSc patients with ATA have a poor prognosis and are more likely to develop serious lung fibrosis ${ }^{21}$. SSc patients with ATA have a higher mortality rate when compared with SSc patients who do not have these autoantibodies $^{12}$. Anti-Th/To RNP is present in $1-13 \%$ of patients with SSc. Anti-Th/To RNP has a strong correlation with the incidence of SSc-ILD.20

A total of $85 \%$ of patients with positive ATA are often affected by lung fibrosis and are protected against isolated pulmonary arterial hypertension, according to Bahmer et al. ${ }^{13}$. In both SSc and SSc-ILD, ATA titers appear to be related to disease severity and activity ${ }^{22}$. In a pilot study, patients, primarily diagnosed as SScILD but with positive and nucleolar-staining ANA, were subsequently tested for $\mathrm{Th} / \mathrm{To}$-autoantibodies and showed positive results $50 \%{ }^{23}$. However, the role of these autoantibodies is unknown.

The second strategy examination (Figure 2) started with Immunoblot, Euroline examination. Out of 42 patients with SSc-ILD, there were 20 patients with ATA autoantibodies, 9 patients with anti-Th/To RNP, 13 patients with non-specific SSc autoantibodies. Then it was continued with IIF ANA examination with a dominant homogenous nucleolar pattern. These 13 non-specific autoantibodies were observed in 6 patients with anti-PM-Scl75, 4 patients with anti-Ro52, and 3 patients with anti-fibrillarin. Anti-PM/Scl autoantibodies have been discovered in a subset of myositis and/or systemic sclerosis patients. 
Anti-PM-Scl antibodies are found in around 25\% of SSc patients with myositis overlap, but only $2 \%$ of SSc patients without myositis overlap ${ }^{12}$. According to Lega et al., anti-PM/Scl patients had symptomatic pulmonary involvement with a favorable prognosis. The characteristics of ILD associated with anti$\mathrm{PM} / \mathrm{Scl}$ autoantibodies have never been described in detail before ${ }^{24}$.

Autoantibodies to Ro52 overlapped with many other autoantibodies. Anti-Ro52 titers were significantly higher in patients with Overlap Syndrome compared to patients without it, according to Hudson et al. ${ }^{10}$. Patients with ILD had significantly higher titers than those without (mean titer, 1,542.65 versus $938.11 \mathrm{U} / \mathrm{ml}$, respectively; $\mathrm{p}=0.06$ ). Several studies demonstrated that anti-Ro52 is present in several systemic autoimmune rheumatic diseases. Anti-Ro52 autoantibodies are commonly found in inflammatory myositis, particularly when anti-Jo1 and interstitial lung disease are present ${ }^{10}$.

The advantage of the first strategy is that it can help diagnose SSc-ILD through SSc-ILD-specific autoantibodies which can be examined through the ANAIIF screening followed by the SSc-Profile examination. The disadvantage of Thoracic HRCT examination as a gold standard that is only available in health facilities in big cities. Specific autoantibody examination does not require sophisticated equipment compared to HRCT examination. The examination of specific autoantibodies is expected to overcome this limitation. In addition to being affordable, it makes examination procedures easier. Another advantage of the examination of specific autoantibodies is in diagnosing SSc-ILD patients with Systemic Sclerosis Sine Scleroderma (ssSSc). SSc-ILD patients with Systemic Sclerosis Sine Scleroderma means that these SSc patients have pulmonary manifestations without skin involvement, so to diagnose SSc in these patients HRCT alone is insufficient and must be supported by specific autoantibodies test.

According to research by Hax et al. in Brazil in 2017, the prevalence of ILD up on HRCT scan was $57.1 \%$. Thoracic HRCT examination alone cannot be used in diagnosing SSc-ILD, it should be supported by clinical symptoms of the patient, physical examination of the patient, laboratory tests that support the diagnosis of SSc-ILD, one of which is autoantibody examination $^{25}$.

In this study, especially the first strategy with better performance, from 42 SSc-ILD patients (who had been examined by HRCT) there were 39 patients with SSc-ILD-specific autoantibodies (nucleolar and nucleolar homogeneous patterns (ANA-IIF) and ATA and Th/To autoantibodies). The first strategy consisting of antinuclear autoantibodies screening (ANAIIF) and followed by autoantibody specific autoantibodies detection (Anti-topoisomerase I, Anti Th/To, SSc profile, Immunoblot) were more likely to detect specific autoantibodies toward ILD in SSc patients.

The second strategy is a specific autoantibody examination (SSc Profile) followed by a screening examination (ANA-IIF). The author's background for this second strategy is the price of the first strategy examination (ANA-IIF followed by SSc Profile) which is still relatively high for SSc patients in Indonesia. The author intends to understand if the second strategy for examination (specifically the SSc Profile) can adequately diagnose SSc-ILD. From the results of this second strategy examination, 13 patients with non-specific SSc-ILD autoantibodies were found. This means that the specific autoantibodies detection modality was not good enough in diagnosing SSc-ILD or maybe the patients had an Overlap Syndrome. Specific autoantibodies detection needs to be initiated by autoantibodies screening examination. The first strategy, which combined autoantibody screening and specific autoantibody detection, revealed more subjects with pulmonary fibrosis than the second strategy. The screening examination (IIFANA) followed by the detection of specific autoantibodies has become the standard in diagnosing SScILD.

The limitations of this study were the small number of subjects. These two laboratory techniques would need further research to be verified.

\section{CONCLUSIONS}

The first strategy consisting of antinuclear autoantibodies screening (ANA-IIF) followed by autoantibody specific autoantibodies detection (Antitopoisomerase I, Anti Th/To, SSc profile, Immunoblot) is more likely to detect autoantibodies specific to ILD in SSc patients.

\section{ABBREVIATIONS}

ACR/EULAR: The American College of Rheumatology/European League against Rheumatism

ANA-IIF: Antinuclear antibodies indirect immunofluorescence

ATA: Anti-topoisomerase-I

ELISA: Enzyme-linked immunosorbent assay

Hep-2: Human epithelial cell line-2

HRCT: High Resolution Computed Tomography

ILD: Interstitial lung disease

MCTD: Mixed Connective Tissue Disease

PFT: pulmonary function test 
SSc: Systemic Sclerosis

SSc Profile: Systemic Sclerosis Profile Test

SSc-ILD: Systemic Sclerosis-related Interstitial Lung Disease

Th/To: anti-Th/ToRNP

\section{ACKNOWLEDGMENTS}

This study was supported by an internal research grant from Universitas Padjadjaran Bandung, West Java, Indonesia.

The autoantibodies screening and specific autoantibodies test used ANA IIF and SSc profile Immunoblot, Euroimmun - Germany.

We thank Dr. Adhi Kristianto Sugianli for his input, ideas, and support until the completion of this research.

\section{AUTHOR'S CONTRIBUTIONS}

Anna Tjandrawati, Sumartini Dewi and Verina Logito had full access to all of the data in the study and takes responsibility for the integrity of the data and the accuracy of the data analysis. Anna Tjandrawati, Verina Logito, Winda Agnestia Maranna Saragih, Andri Reza Rahmadi, and Sumartini Dewi contributed substantially to the study design, data analysis and interpretation. Anna Tjandrawati, Sumartini Dewi, and Verina Logito was the primary author and Verina Logito was a major contributor in writing the manuscript. All authors read and approved the final manuscript.

\section{FUNDING}

This study was supported by an internal research grant from Universitas Padjadjaran Bandung, West Java, Indonesia.

\section{AVAILABILITY OF DATA AND MATERIALS}

On reasonable request, the corresponding author will provide the data used and/or analyzed during the current analysis.

\section{ETHICS APPROVAL AND CONSENT TO PARTICIPATE}

The institutional review board of the Faculty of Medicine Universitas Padjadjaran / Dr. Hasan Sadikin General Hospital Bandung, Indonesia approved the protocol \#2392020 entitled "Pengembangan Sistem Registri SSc berbasis Electronic Medical Record". All patients gave their informed consent to participate in the study, which was approved by our local Ethics Committee of Faculty of Medicine Universitas Padjadjaran / Dr. Hasan Sadikin General Hospital Bandung, Indonesia.

\section{CONSENT FOR PUBLICATION}

Not applicable.

\section{COMPETING INTERESTS}

Anna Tjandrawati is an academic author and the Head of Division Immunoserology, Department of Clinical Pathology, Faculty of Medicine Padjadjaran University. Sumartini Dewi is an academic author, an Internist, Rheumatologist in Division of Rheumatology, Department of Internal Medicine, The Head of Immunology Study Center, Faculty of Medicine Padjadjaran University. Andri Reza Rahmadi is an academic author, an Internist, Rheumatologist in Division of Rheumatology, Department of Internal Medicine, Faculty of Medicine Padjadjaran University. The remaining authors declare no relevant financial disclosures or conflicts of interest.

\section{REFERENCES}

1. van den Hoogen F, Khanna D, Fransen J, Johnson SR, Baron M, Tyndall A. 2013 classification criteria for systemic sclerosis: an American College of Rheumatology/European League against Rheumatism collaborative initiative. Arthritis and Rheumatism. 2013;65(11):2737-47. PMID: 24122180. Available from: 10.1002/art.38098.

2. Katsumoto TRWM, Connolly MK. The Pathogenesis of Systemic Sclerosis. Annu Rev Pathol Mech Dis. 2011;6:509-537. Available from: 10.1146/annurev-pathol-011110-130312.

3. Khanna D, Tseng CH, Farmani N, Steen V, Furst DE, Clements PJ. Clinical course of lung physiology in patients with scleroderma and interstitial lung disease: analysis of the Scleroderma Lung Study Placebo Group. Arthritis and Rheumatism. 2011;63(10):3078-85. PMID: 21618205. Available from: 10.1002/art.30467.

4. Mirsaeidi M, Barletta P, Glassberg MK. Systemic sclerosisassociated interstitial lung disease: new directions in disease management. Frontiers in Medicine. 2019;6:248. PMID: 31737640. Available from: 10.3389/fmed.2019.00248.

5. Barnes J, Mayes MD. Epidemiology of systemic sclerosis: incidence, prevalence, survival, risk factors, malignancy, and environmental triggers. Current Opinion in Rheumatology. 2012;24(2):165-70. PMID: 22269658. Available from: 10.1097/ BOR.0b013e32834ff2e8.

6. Budiman AM, Dewi S, Prananta MS. Clinical Manifestation and Laboratory Finding of Sclerosis Systemic Patient in Dr. Hasan Sadikin General Hospital Bandung: A Descriptive Quantitative Study. Indonesian Journal of Rheumatology. 2018;10(1):1519. Available from: 10.32539/ijr.v10i1.93.

7. Solomon JJ, Olson AL, Fischer A, Bull T, Brown KK, Raghu G. Scleroderma lung disease. European Respiratory Review. 2013;22(127):6-19. Available from: 10.1183/09059180. 00005512.

8. Cottin V, Brown KK. Interstitial lung disease associated with systemic sclerosis (SSc-ILD). Respiratory Research. 2019;20(1):13. PMID: 30658650. Available from: 10.1186/ s12931-019-0980-7.

9. Goh NS, Desai SR, Veeraraghavan S, Hansell DM, Copley SJ, Maher TM. Interstitial lung disease in systemic sclerosis: a simple staging system. American Journal of Respiratory and Critical Care Medicine. 2008;177(11):1248-54. PMID: 18369202. Available from: $10.1164 / \mathrm{rccm} .200706-877$ OC. 
10. Hudson M, Pope J, Mahler M, Tatibouet S, Steele R, Baron M, et al. Clinical significance of antibodies to Ro52/TRIM21 in systemic sclerosis. Arthritis Research \& Therapy. 2012;14(2):50. PMID: 22394602. Available from: 10.1186/ar3763.

11. Takei R, Arita M, Kumagai S, Ito Y, Tokioka F, Koyama T. Radiographic fibrosis score predicts survival in systemic sclerosisassociated interstitial lung disease. Respirology (Carlton, Vic). 2018;23(4):385-91. PMID: 28925574. Available from: 10.1111/ resp. 13175.

12. Autoantibodies YH. Autoantibodies in Systemic Sclerosis. In: Takehara K., Fujimoto M., Kuwana M. (eds) Systemic Sclerosis. Springer Tokyo: 2016 pp 231-247;.

13. Bahmer T, Romagnoli M, Girelli F, Claussen M, Rabe KF. The use of auto-antibody testing in the evaluation of interstitial lung disease (ILD) - A practical approach for the pulmonologist. Respiratory Medicine. 2016;113:80-92. PMID: 26921132 Available from: 10.1016/j.rmed.2016.01.019.

14. Varga JL. Etiology and pathogenesis of systemic sclerosis. Rheumatology. Philadelphia: Elsevier; 2015.

15. Kayser C, Fritzler MJ. Autoantibodies in systemic sclerosis: unanswered questions. Frontiers in Immunology. 2015;6:167. PMID: 25926833. Available from: 10.3389/fimmu.2015.00167.

16. Walker JG. Systemic Sclerosis. In: Diagnostic Criteria in Autoimune Diseases. New York: Humana press; 2008: p. 31. Available from: 10.1007/978-1-60327-285-8_6.

17. Bergamasco A, Hartmann N, Wallace L, Verpillat P. Epidemiology of systemic sclerosis and systemic sclerosis-associated interstitial lung disease. Clinical Epidemiology. 2019;11:25773. PMID: 31114386 . Available from: 10.2147/CLEP.S191418.

18. Steen VD. Autoantibodies in systemic sclerosis. Semin Arthritis Rheum. 2005;35(1):35-42. PMID: 16084222. Available from: 10.1016/j.semarthrit.2005.03.005.

19. McNearney TA, Reveille JD, Fischbach M, Friedman AW, Lisse JR, Goel N. Pulmonary involvement in systemic sclerosis: as- sociations with genetic, serologic, sociodemographic, and behavioral factors. Arthritis and Rheumatism. 2007;57(2):318 26. PMID: 17330281 . Available from: 10.1002/art.22532.

20. Cappelli S, Randone SB, Camiciottoli G, Paulis AD, Guiducci $S$, Matucci-Cerinic M. Interstitial lung disease in systemic sclerosis: where do we stand? European Respiratory Review. 2015;24(137):411-9. PMID: 26324802. Available from: $10.1183 / 16000617.00002915$.

21. Yamakawa $H$, Hagiwara E, Kitamura $H$, Yamanaka $Y$, Ikeda $S$ Sekine A. Clinical features of idiopathic interstitial pneumonia with systemic sclerosis-related autoantibodies in comparison with interstitial pneumonia with systemic sclerosis. PLoS One. 2016;11(8):e0161908. PMID: 27564852. Available from: 10 1371/journal.pone.0161908.

22. Hu PQ, Fertig N, Medsger TA, Wright TM. Correlation of serum anti-DNA topoisomerase I antibody levels with disease severity and activity in systemic sclerosis. Arthritis and Rheumatism. 2003;48(5):1363-73. PMID: 12746909. Available from: 10.1002/art.10977.

23. Fischer A, Pfalzgraf FJ, Feghali-Bostwick CA, Wright TM, Curran-Everett D, West SG. Anti-th/to-positivity in a cohort of patients with idiopathic pulmonary fibrosis. The Journal of Rheumatology. 2006;33(8):1600-5. PMID: 16783860.

24. Lega JC, Cottin V, Fabien N, Thivolet-Béjui F, Cordier JF. Interstitial lung disease associated with anti-PM/Scl or antiaminoacyl-tRNA synthetase autoantibodies: a similar condition? The Journal of Rheumatology. 2010;37(5):1000-9. PMID 20231208. Available from: 10.3899/jrheum.090652.

25. Hax V, Bredemeier M, Moro AL, Pavan TR, Vieira MV, Pitrez EH, et al. Clinical algorithms for the diagnosis and prognosis of interstitial lung disease in systemic sclerosis. InSeminars in arthritis and rheumatism. 2017;47(2):228-234. Available from: 10.1136/annrheumdis-2017-eular.1448. 
Ready to submit your manuscript? Choose Biomedpress and benefit from:

- Fast, convenient online submission

- Through peer-review by experienced researchers

- Rapid publication on acceptance

- Free of charge (without publication fees)

Learn more http://www.biomedpress.org/journals/

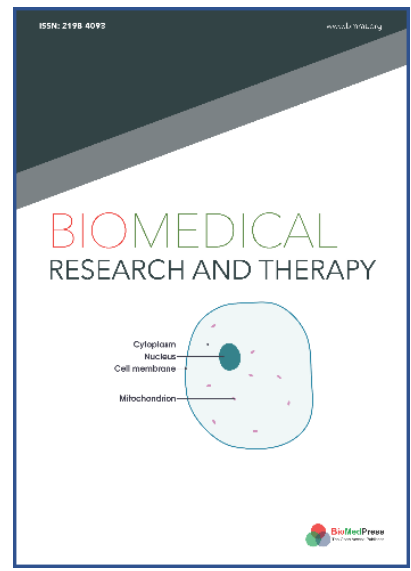

\title{
Biomedical Research and Therapy
}

Indexed: Web of Science (ESCl), Embase, Google Scholar

Journal Citation Indicator (2020): 0.16

Acceptance Rate (2020): 54.32\%

Article Publishing Charge: Free

Submission to first editorial decision: 27 days

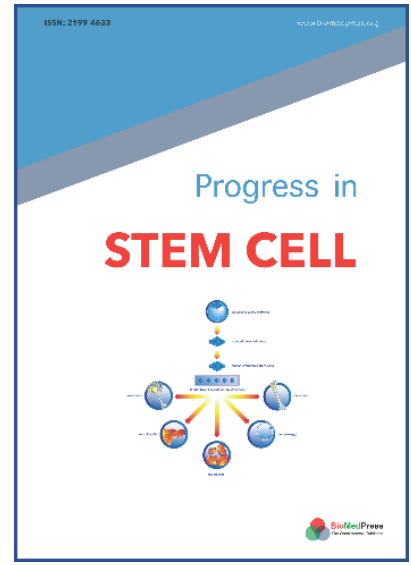

\section{Progress in Stem Cell}

Indexed: Embase, Google Scholar

Acceptance Rate (2020): 78.19\%

Article Publishing Charge: Free

Submission to first editorial decision: 19 days

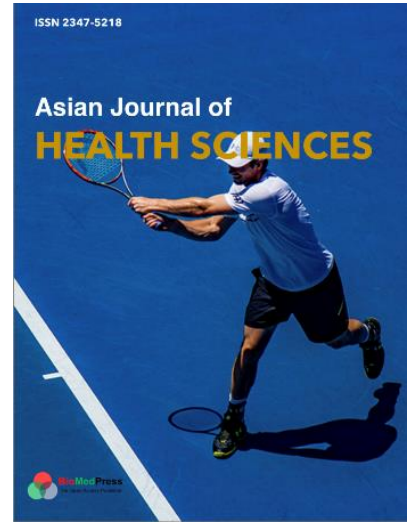

\author{
Asian Journal of Health Sciences \\ Indexed: Google Scholar \\ Acceptance Rate (2020): 72.89\% \\ Article Publishing Charge: Free \\ Submission to first editorial decision: 16.5 days
}

